\title{
Negociación digital Internacional y sus procesos
}

Jenniffer Sobeida Moreira Chóez

jenniffer.moreira@utm.edu.ec https://orcid.org/0000-0001-7172-7459

Universidad Técnica de Manabí

Veronica Monserrate Mendoza Fernandez

Veronica.mendoza@utm.edu.ec https://orcid.org/0000-0002-2937-4052

Universidad Técnica de Manabí

Portoviejo - Ecuador

\section{RESUMEN}

Las herramientas digitales en el proceso de negociación internacional han provocado cambios importantes en la mesa de negociación, haciendo que la relación contractual sea más ágil y eficiente. Este artículo científico se enfoca en identificar los 6 tipos de negociaciones como: distributiva, integrativa, colaborativa, explícita, tácita y por posición, donde cada una de ellas cumple un papel importante en la negociación, así mismo, los procesos de la negociación digital internacional como la preparación, diálogo, acuerdo y cierre, los cuales son fundamentales para cumplir con el propósito de satisfacer las necesidades de la empresa. Por lo antes mencionado, el objetivo de esta investigación es realizar un análisis de las negociaciones digitales y sus procesos. La investigación es de tipo analítica y cuya metodología se basa en referencias literarias mediante análisis documental de diferentes revistas y libros de bases de datos de alto impacto, que permiten dar soporte sustentable al marco teórico de la presente investigación.

Palabras clave: Negociación; Negociación digital Internacional; TIC; procesos; relación contractual 


\title{
International digital negotiation and its processes
}

\begin{abstract}
Digital tools in the international negotiation process have caused important changes at the negotiation table, making the contractual relationship more agile and efficient. This scientific article focuses on identifying the 6 types of negotiations such as: distributive, integrative, collaborative, explicit, tacit and by position, where each one of them plays an important role in the negotiation, likewise, the processes of international digital negotiation such as preparation, dialogue, agreement and closing, which are fundamental to fulfill the purpose of satisfying the needs of the company. Due to the aforementioned, the objective of this research is to carry out an analysis of digital negotiations and their processes. The research is analytical and whose methodology is based on literary references through documentary analysis of different magazines and high-impact database books, which allow to give sustainable support to the theoretical framework of this research.
\end{abstract}

Keywords: Negotiation; International digital negotiation; TIC; processes; contractual relationship

Artículo recibido: 05 de Abril 2021

Aceptado para publicación: 28 de Mayo 2021

Correspondencia: jenniffer.moreira@utm.edu.ec

Conflictos de Interés: Ninguna que declarar 


\section{INTRODUCCIÓN}

La negociación es quizás la actividad principal de la diplomacia internacional contemporánea. Es el mecanismo central a través del cual las naciones resuelven problemas comunes y conflictos internacionales, ya sea en la forma de negociaciones multilaterales formales, o a través de intercambios bilaterales informales (Hopmann, 1990, pág. 7).

El término empleado en negociación internacional es confuso porque los autores, tanto de medios masivos como de informes gubernamentales y académicos los usan para definir diferentes cosas (Daniels, Radebaugh, \& Sullivan, 2004), sin embargo, sostienen que la negociación internacional abarca un crecimiento constante alrededor del mundo, en la actualidad las competencias afectan tanto a la macro y micro empresas, ya que en su totalidad venden productos a países extranjeros y adquieren suministros de ellos.

Por otra parte, la negociación internacional es aislada de la negociación que se realiza entre empresas de un mismo territorio, debido a muchos factores como: marco legal, diferencia de idioma, cultura y protocolo en la negociación. Por ello, que la existencia de las Tecnologías de la Información y Comunicación (TICs) contribuyen a la construcción y el desarrollo de estos procesos.

El proceso de negociación internacional se define como un conjunto de actividades a través de etapas o fases como la preparación, discusión, acuerdo, cierre y seguimiento de la negociación, permiten que se realicen operaciones o actividades de importación y exportación en el ámbito internacional. Para Cano \& Baena (2015) el proceso de negociación se origina con las necesidades y demandas que identifica la empresa en la compraventa de bienes o servicios de forma cosmopolita, y concluye con la generación de un acuerdo o contrato que legaliza el vínculo comercial entre ambas partes.

Es evidente entonces, que las TIC facilitan la construcción de relaciones, la gestión de información y el planteamiento de alternativas de negociación ((Baena et al., 2014), Adicional a esto, las herramientas digitales en el proceso de negociación internacional presentan varios factores que influyen en el aumento eficiente de la economía, es decir, en ahorros de gastos que se obtienen por traslado y movilidad. Del mismo modo, permite acelerar volúmenes de información y optimizar las diversas actividades administrativas realizadas por la organización. 
Las herramientas digitales han sido el gran aliado en este proceso de negociación, convirtiéndose en un intermediario en la relación comercial de los diferentes países en todo el mundo. La incorporación de las (TIC) ha contribuido en el desarrollo de las organizaciones, siendo un instrumento importante para los procesos operativos de las empresas.

Varios investigadores alrededor del mundo, han tomado como objeto de estudios la incorporación de las herramientas digitales en la negociación, cuyos estudios destacan a las herramientas tecnológicas como Thompson \& Nadler (2002) se enfocan en el correo electrónico, teléfono y fax como sistema de negociación electrónica, por su parte, Cano \& Baena (2013), sostienen que las TIC pueden influir en el comportamiento comercial internacional de las empresas debido que cada vez más utilizan estas herramientas digitales para reducir costos y tiempo en los contratos con otras empresas, así mismo, permite estandarizar sus procesos de forma optima en las operaciones de compraventa internacional.

El presente artículo tiene como objetivo identificar, a través de revisiones literarias las negociaciones digitales Internacional y sus procesos. Partiendo de este apartado se presentan en la metodología las fuentes de información consultadas para sustentar el artículo, que por su contexto permitieron obtener una mejor comprensión de las influencias de las TIC en la negociación internacional. Luego, en el marco teórico se describe la conceptualización de la negociación, diferencia entre negociación tradicional y digital, tipos de negociaciones, procesos de negociación internacional y la negociación digital internacional.

\section{METODOLOGÍA}

Para la búsqueda de información del marco teórico se acudió principalmente a bases de datos de revistas científicas como SCOPUS, SCIELO y EBSCO, GOOGLE ACADÉMICO, entre otros, en las cuales se realizó búsqueda de artículos científicos que tuvieran dentro del título, resumen o palabras clave, conceptos y términos tales como: Negociación, Negociación Internacional, tecnologías de información y comunicación, entre otros. $\mathrm{Al}$ respecto, las conceptualizaciones y términos de búsqueda realizadas en las bases de datos se ingresaron tanto en español como inglés. Por consiguiente, para la información bibliográfica del preste trabajo investigativo fue realizada con fichas bibliográficas en formato Excel, que permitió obtener las referencias bibliográficas de 
forma organizada, resumen de cada artículo, métodos y resultados obtenidos que permitirán sustentar las conceptualizaciones de la negociación digital internacional y sus procesos, con el fin de dar soporte a esta investigación.

\section{MARCO TEÓRICO}

En el Marco Teórico se abordará la definición de negociación, así mismo, los tipos de negociaciones y sus procesos, posteriormente se abarcará influencia de las TIC en el proceso de negociación internacional y su importancia de las herramientas digitales en este proceso, generando un sustento teórico para finalizar con la luego profundizaren la caracterización de estas tecnologías.

\section{Definición de negociación}

Desde el inicio de nuestra existencia el ser humano ha tenido la necesidad de comunicarse unos con otros, para obtener algún beneficio o satisfacer sus necesidades básicas para su sobrevivir, como alimento, vestimenta, entre otros; así mismo, la negociación fue incorporada para el trueque de alimentos y el comercio, que, a su vez, el precio para la compra y venta era acordado mediante la persuasión.

La palabra negociación para Real Academia Española (2014), define estos conceptos de la siguiente manera "mediante la negociación se ajusta el traspaso, cesión o endoso de un vale, de un efecto o de una letra o en otras palabras a través de la negociación se procura la mejor solución de las disputas de asuntos de derecho público o privado. La negociación es la acción y efecto de negociar. También es posible definir a la negociación como los tratos dirigidos a la conclusión de un convenio o pacto", por su parte, Hopmaun et al., (1990) citando a William Zartman, sostiene que la negociación son procesos básicos, dinámico y no una situación estática para tomar una decisión, es ahí donde debe seleccionar diversos valores para su implementación y acción para llegar a una resolución. Es importante entender que un administrador, gerente o ejecutivo debe tener buenas capacidades y habilidades para negociar, ya que esta técnica ha demostrado que el principal papel de los administradores es mantener negociaciones con diferentes grupos o empresas como representante de la organización, y cuyo rol tiene una valiosa connotación interna, para intervenir como mediador en una disputa o negociar entre áreas. Por ello, a la negociación se la define como un proceso que participan dos o más personas con el fin de intercambiar o conseguir algún beneficio que les permita crear, ganar ventajas, obtener, mantener relaciones comerciales. 
Ahora bien, se puede definir a la negociación como el rumbo de las necesidades en los diferentes entornos, hasta la satisfacción de ambas partes mediante un acuerdo que implica compromiso, responsabilidad y beneficios para ambos grupos.

\section{Negociación tradicional + Negociación digital}

La negociación tradicional Sanchez \& Fernández (2020), las partes intervinientes trabajan en modo de negociar por posiciones, empatía con el fin de buscar un acuerdo win-win y cuyo modelo tradicional es el arte de negociar por medio de los procesos adaptativos, consultivo y transformacional, siedo identificado en los años 1900 por Rosann L. Spiro y Barton A. Weitz, quienes los definian como habilidades para cambiar o modificar sus comportamientos en las relaciones que mantinen con sus clientes por la percepciones e información percibida en una situación comercial.

Por otra parte, la negociación digital es la transformación de la negociación tradicional, en este proceso se negocia estratégicamente utilizando herramientas que utilizan lenguaje digital disponibles en el internet como publicaciones, correos electrónicos, foros, banco de datos, instrumentos audiovisuales y plataformas virtuales que permiten comunicarse de forma sincrónica y asincrónica con los usuarios o proveedores.

Por lo tanto, toda negociación involucra varias formas de interacción y relación entre grupos de individuos o empresas. Es decir, esta acción es la combinación entre el tipo de negociación digital y la tradicional. Por lo tanto, el objetivo de la negociación digital no es eliminar la negociación tradicional, por el contrario, la unión de ambas constituye el equilibrio perfecto para una negociación optima y eficaz en un proceso contractual, generando así el sustento teórico de la presente investigación.

\section{Tipos de negociación}

Los tipos de negociación según García (2014) son en base a las diferentes actitudes que toman las partes en el momento de la negociación y cuyas relaciones contractuales dependerán de la forma de negociar de ambos grupos de sus actitudes, habilidades y estrategias de los negociadores. Sin embargo, Sánchez \& Fernández (2020), sostienen que los tipos de negociación se hacen referencia de acuerdo como se los plantea ambas partes siendo estos luchar o cooperar.

De acuerdo a las consideraciones anteriores y luego de haber realizado una amplia busqueda de los tipos de negociaciones, es importante dar a conocer los tipos de negociaciones más relevantes. 
Figura 1. Tipos de negociaciones

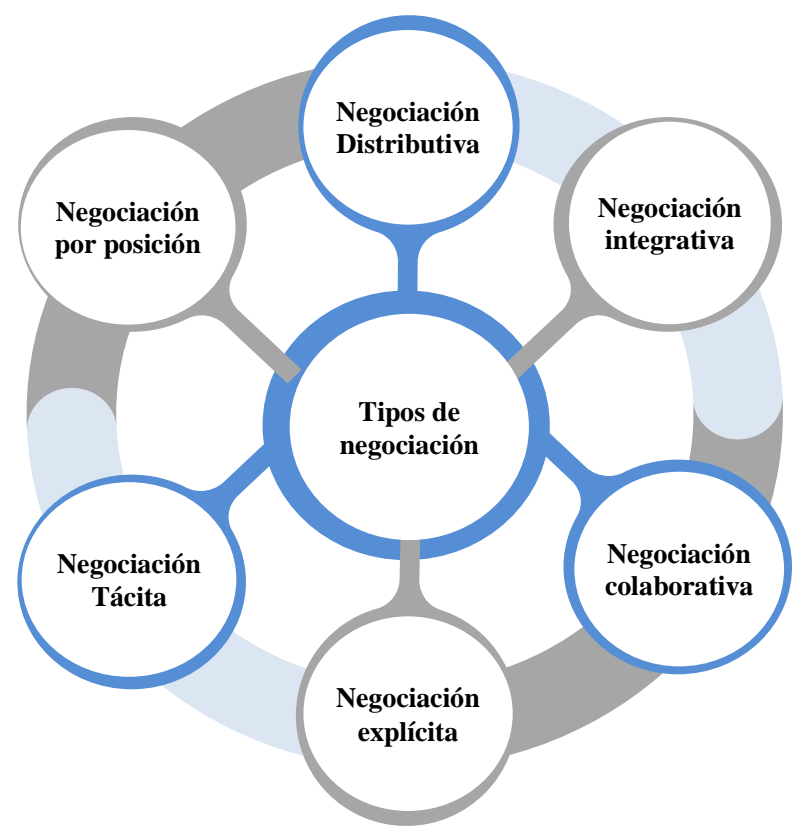

Fuente: Elaboración propia

La negociación distributiva, se produce cuando los recursos son limitados, simultáneamente con magnitudes opuestas, como el tiempo, precio, entre otros. Por lo tanto, los integrantes de la negociación consideran como se debe distribuir los recursos y utilizan diferentes técnicas y estrategias para conseguir el mayor beneficio en este proceso, donde ambos colectivos entran en discusión o regateo para alcanzar el mejor resultado posible (Sanchez \& Fernández, 2020). Este tipo de relación contractual prima la desconfianza entre ambas partes y se protege celosamente la información, es básicamente una competencia sobre quien obtendrá mejor beneficio y que una de partes alcance sus objetivos, dependerá de las estrategias y tácticas que emplee en este proceso. Por otra parte, la negociación Integrativa, es la que ofrece metas que no crearán un conflicto directo con los objetivos fundamentales del otro (Hopmann, 1990). En este proceso de negociación las partes negociadoras se enfocan en cumplir sus intereses y necesidades y de la otra parte con el propósito de generar una relación contractual que permita alcanzar los objetivos de forma conjunta para generar beneficios mutuos. Para ello, es necesaria la integración de las herramientas digitales en esta fase de negociación con el propósito de agilitar el marco común que es la resolución definitiva de la negociación. 
Esta técnica es importante para cualquiera que participe en una negociación. A menos que una de las partes sepa cómo reclutar a la otra en un descubrimiento mutuo de intereses, el comportamiento negociador normalmente resultará en una ronda de negociación "de suma cero" o "distributiva" mutuamente derrotada (Keiser University, 2020). De esta manera, la integratividad en la negociación representa el interés de ambas partes es evitar el cierre del contrato o acuerdo siempre y cuando exista que ambos colectivos lleguen a una satisfacción en común.

Otro factor importante en un acuerdo es la negociación colaborativa, (Thomas et al., 2013) consideran que en el ámbito colaborativo el enfoque de la negociación es ganar ganar aumenta la relación contractual con el proveedor a largo plazo permitiendo que esta relación exista intercambio de información, confianza y traspaso de cultura organizacional. Por su parte, (Lozano et al., 2011) la colaboración entre usuarios en el documento base es posible gracias a la interfaz de la herramienta y a la característica de edición multiusuarios que muestra una etiqueta con un color y el nombre para identificar y tener un seguimiento en tiempo real de quien está escribiendo, que se está escribiendo, que se está cambiando y aportando al documento que se torna colectivo (pág. 25).

La Negociación explícita es también denominado formal, o manifestación consciente de la negociación, se da cuando la relación es conceptualizada como un intercambio explícito de oferta y contraofertas en el que las acciones de hallan conscientemente diseñadas para encontrar una solución mutuamente aceptable del conflicto (Munduate Jaca , 1987, pág. 31). Es decir, en este proceso de negociación los mediadores son conscientes de que están negociando.

Por consiguiente, en la negoción tácita las partes no son conscientes de que están negociando, pero quieren solucionar la disidencia. Es decir, que las partes tienen problemas para comunicarse, por lo tanto, no definen la relación contractual, (Gonzalez García, 2006 ) sostienes que la existencia de esta negociación es porque a veces la propia negociación explícita no llega a producirse en una relación contractual, por lo tanto, esta negociación influye tanto o más en los resultados de una negociación.

Por otra parte, en la negociación por posición cada colectivo toma una posición al inicio de la relación contractual, en esta fase inician con el dialogo o discusión por medio de las TIC para mantener su posición defensiva sobre los intereses económicos o beneficios que desea adquirir la parte negociadora. Además, es considerada la negociación por posición, 
como un proceso competitivo, donde se suelen conseguir los acuerdos de manera rápida sin considerar a la otra parte.

Cabe indicar, que las tecnologías tienen cada vez más participación en cada una de las fases del proceso de negociación internacional, principalmente aquellas herramientas digitales que pueden reemplazar actividades y operaciones habituales, y las que disponen una amplia integración y conectividad con otras tecnologías.

\section{Negociación Digital Internacional}

En una relación contractual es indudable que existe un ambiente donde las partes involucradas buscan cumplir y satisfacer sus objetivos y necesidades a partir de la prerrogativa que estas puedan conceder, sin embargo, es imprescindible que se encuentren alternativas que permitan que todo el proceso de negociación sea eficiente y cumpla con el objetivo.

Desde esta perspectiva se construye la polémica sobre que es más conveniente y optimo en una relación contractual, particularmente en una etapa de compra y venta internacional, donde ambas partes no se conocen de forma física y donde la confianza se obtiene cuando las partes interactúan y conocen su entorno, tanto como aspectos personales y comerciales. Es en tal punto cuando la definición de la negociación se hace manifiesto, debido a que los socios de la organización y sus representantes legales, mantienen conductas diferentes en el momento de negociar; esto con el pasar de los años y las tendencias como las TIC ha obtenido un cambio evidente, donde existen diversas opciones que brinda esta herramienta digital para realizar acuerdos y mantener la relación comercial con los clientes y proveedores, sean estas por chat, video llamadas, correo electrónico, entre otros.

\section{Procesos de negociación digital Internacional}

La alianza estratégica es compleja en toda negociación Internacional. La gran parte de las organizaciones envían como intermediarios en la negociación abogados o consultores para que actúen como asesores debido a los procesos legales que se efectúan en la relación contractual. El aspecto importante en este tipo de negociación el que tiene mayor control es el socio mayoritario de la empresa, sin embargo, es esencial dar apertura a la contra parte para que explique los beneficios que se van adquirir en este acuerdo, para ello es necesario conocer las diferentes etapas de la negociación. 
Es por ello, que a los procesos de negociación se lo define como un conjunto de fases donde los negociantes buscan satisfacer las necesidades de la empresa, con el fin de obtener beneficios, compromisos y acuerdos ventajosos para ambos sectores comerciales. De acuerdo a las consideraciones anteriores, para el proceso de negociación se debe tener en cuenta 5 etapas o fases como: Preparación, diálogo, Acuerdo y Cierre.

Figura 2. Procesos de negociación Internacional

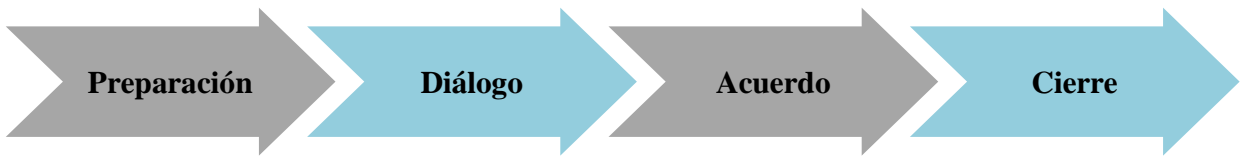

Fuente: Elaboración propia

Preparación: En esta fase el negociador debe tomar el control, dirigir, elegir como responder y actuar en el proceso de negociación, es aquí donde se ponen en claro las normas y reglas de negociación, aunque, cada negociante debe tener claro sus metas y objetivos, así mismo, que información debe proporcionar y cual no, siempre manteniendo los límites de acuerdo a lo acordado por la empresa que va a negociar, por otra parte, es importante obtener información de la parte contraria, con el propósito de obtener una visión para implementar estrategias al negociar.

Es importante que el negociador en esta etapa, no sea el protagonista de la negociación ya que el protagonista del proceso es el cliente, por lo tanto, el negociador debe escuchar primeramente a la contraparte con el fin de llegar en un acuerdo para ambas partes.

Diálogo: El diálogo es la etapa central y la más importante en el proceso de negociación. Inicia desde que empieza la conversación entre ambas partes en la mesa de negociación hasta que se concluye el proceso de negociación sea esta de manera tradicional o digital. En esta fase se trata de conseguir o acercar a un acuerdo final, por medio de la persuasión o dialogo con el propósito de llegar al objetivo que es obtener una relación contractual para satisfacer las necesidades de ambos grupos.

Esta etapa de negociación actualmente, se la realiza por medio de las herramientas digitales las cuales cumplen un rol importante es este proceso cuando las partes se encuentran en diferentes países o territorios. Sin embargo, para que sea exitosa la negociación debe existir respeto, empatía, confianza, flexibilidad, creatividad, 
asertividad, paciencia entre otros factores que permitirán que este proceso sea favorable para ambas partes.

Acuerdo: En todo proceso de negociación el objetivo principal es llegar un acuerdo entre ambas partes, una vez que se ha realizado la preparación y la discusión de la negociación la etapa del acuerdo es una de la más importante para el negociador, donde se sustenta por escrito las normas jurídicas y acuerdos de la negociación utilizando las TIC para su desarrollo, con el fin de reducir riegos o cambio de opinión de la contraparte, en este proceso se plasma los puntos tratados y datos específicos de la negociación.

Obtener un acuerdo en la negociación, permitirá marcar la sostenibilidad de la empresa, ya que debido a este proceso se generará automáticamente un campo de rentabilidad que será de enlace para futuras negociaciones.

Cierre: Una vez realizado el acuerdo entre ambas partes se procede con la finalización de la negociación, en este proceso se debe analizar con sentido crítico como se llevó a cabo este proceso, con el propósito de encontrar aquellos desfases que se deben mejorar para futuras relaciones contractuales.

La negociación se concluye luego de haber empleado diversas técnicas, estrategias y habilidades, con la intersección de las TIC asociadas a Internet que facilitan la ejecución de transmisión de la información (Stavrovski, 2004), la negociación comercial llega a su fin cuando se la efectúa un contrato. Sin embargo, esta etapa de negociación internacional se puede iniciar con la aplicación de las herramientas digitales con el fin de agilizar el proceso de negociación, el mismo que dependerá de las necesidades y preferencias de la empresa. Por su parte, Cano \& Baena (2013) sostienen que las TIC son herramientas que mejoran notoriamente las relaciones contractuales de las empresas que incurcionan en el proceso de importación y exportación de bienes y servicios.

\section{CONCLUSIÓN}

La negociación es una habilidad que la tiene todo ser humano, sin embargo, algunos la desarrollan y otros no. Este proceso de negociación ha tenido su evolución con el pasar del tiempo desde su conceptualización hasta los diferentes tipos y procesos de negociación internacional.

La presente investigación muestra desde el marco teórico como definen varios autores la negociación, como la Real Academia de la Lengua Española hasta varios investigadores que sostienen sus conceptualizaciones, donde enfatizan que la negociación son procesos 
básicos, dinámico y no una situación estática para la toma de decisiones donde se seleccionan diversa acción para llegar a una resolución.

Otro aspecto importante, es la relación entre negociación tradicional versus la digital, donde ambas cumplen un rol sustancial en el proceso de negociación internacional; sin embargo, la unión de estos tipos de negociaciones permitirá que todo proceso contractual sea más eficiente y eficaz, debido a que la negociación tradicional se la realiza de forma directa con las partes involucradas y la negociación digital interviene los medios tecnológicos como las TIC.

Por otra parte, son considerados los tipos de negociación como actitudes y habilidades de los grupos negociadores que poseen al momento de mantener un acuerdo. Por lo tanto, la presente investigación se centra en 6 tipos de negociaciones como: Distributiva, integrativa, colaborativa, explícita, tácita y por posición, donde cada una de ellas cumple un papel importante en la relación contractual acompañada de las herramientas digitales. Por último, para que toda negociación sea viable es importante tener en consideración las 5 etapas como: Preparación, diálogo, Acuerdo y Cierre, los cuales permitirán que este proceso sea organizado y cumpla con el propósito de satisfacer las necesidades de la empresa. Sin embargo, estas etapas deben estar relacionadas con las TIC, cuyas herramientas son utilizadas para que la negociación internacional sea más eficiente, por la agilidad de negociar, la reducción de tiempo en la negociación y ahorros en gastos de movilidad que permiten mejorar las relaciones contractuales.

\section{Referencias Bibliográficas}

Baena Rojas, J. J., Cano Arenas, J. A., Jarrin Quintero, J. A., \& Pérez Arroyave, H. R. (2014). Uso de Tecnologías de información y Comunicación para la negociación interncacional. ¿Ventajas para las empresas colombianas? Revista Ciencias Estratégicas, 22(32), 279-294. Obtenido de http://www.redalyc.org/articulo.oa?id=151339264007

Cano , J. A., \& Baena , J. J. (2015). Impacto de las tecnologías de información y comunicación en el desempeño de la negociación internacional. REVISTA BRASILEIRA DE GESTÃO DE NEGÓCIOS, 751-768.

Cano Arenas, J. A., \& Baena Rojas, J. J. (2013). Retos en la implementación de las TIC para el proceso de negociación internacional. Scielo, 153 - 163. 
Daniels, J., Radebaugh, L., \& Sullivan, D. (2004). Negocios Internacionales, Ambientes y operaciones. México: PEARSON EDUCACION.

García Lomas, O. L. (2014). Negociación Internacional. Madrid: GlobalMarketing.

Gonzalez García, M. J. (2006 ). Técnicas de Negociacicón. Malaga, España: Innovación y Cualificación S.L.

Hopmann, P. (1990). TEORIA Y PROCESOS EN LAS NEGOCIACIONES INTERNACIONALES. Chile: PNUD - CEPAL.

Hopmaun, T., Lewis, D. J., \& Wynia, G. (1990). TEORIA Y PROCESOS EN LAS NEGOCIACIONES INTERNACIONALES. Suiza: Centre for Applied Studies in International Negotiations.

Keiser University. (14 de Agosto de 2020). Latin American Campus. Obtenido de https://keiseruniversity.edu.ni

Libreros, E., Núñez, Á., Bareño, R., García del Poyo, R., Gutierrez Ulecia, J., \& Pino, G. (2014). El libro de Marketing interactivo y la publicidad digital. Madrid: ESIC Editorial.

Lozano Rodríguez, A., Valdes Lozano, D. E., Sánchez Aradillas, A. L., \& Esparza Duque, E. (2011). USO DE GOOGLE DOCS COMO HERRAMIENTA DE CONSTRUCCIÓN COLABORATIVA TOMANDO EN CUENTA LOS ESTILOS DE APRENDIZAJE. Revista Estilos de Aprendizaje, 4(8), 23-39.

Munduate Jaca , L. (1987). El proceso de negociación en las organizaciones. Revista andaluza de trabajo y bienestar social, 12, 25-47.

Real Academia Española (RAE). (2014). Diccionario de la lengua española. Madrid, España.

Sanchez, P., \& Fernández, R. (2020). Negociación Comercial hacia la Slowbalisation. Madrid: ESIC EDITORIAL.

Stavrovski, B. (2004). Diseño de un nuevo modelo de comercio electrónico para una empresa inmobiliaria comercial: un estudio de caso. Scopus, 28(2), 110-119. 
Thomas, S. P., Thomas, R. W., Manrodt, K. B., \& Rutner, S. M. (2013). Una prueba experimental de los efectos de la estrategia de negociación sobre las intenciones de compartir conocimientos en las relaciones entre compradores y proveedores. Journal of Supply Chain Management, 49(2), 96-113.

Thompson, L., \& Nadler, J. (2002). Negotiation via Information Technology: Theoryand aplication. Journal of Social Issues, 109-124. 